\title{
Viral hemorrhagic septicemia virus alters turbot Scophthalmus maximus macrophage nitric oxide production
}

\author{
C. Tafalla, A. Figueras, B. Novoa* \\ Instituto de Investigaciones Marinas, CSIC, Eduardo Cabello, 6, 36208 Vigo, Spain
}

\begin{abstract}
The effect of viral hemorrhagic septicemia virus (VHSV) in vitro infection on the nitric oxide (NO) production by turbot Scophthalmus maximus kidney macrophages has been addressed in the past. Previously, we had determined that only a small fraction of turbot possess head kidney macrophages that respond to a single exposure of lipopolysaccharide (LPS) with NO production (LPS-responsive macrophages), whereas macrophage cultures from other individuals were not activated by LPS alone and needed a combination of stimuli to respond (LPS-non-responsive macrophages). In the current work, we examined the effect of VHSV on NO production by macrophages characterized as LPS-responsive macrophages or LPS-non-responsive macrophages. Combinations of LPS and tumor necrosis factor $\alpha$ (TNF- $\alpha$ ) and macrophage-activating factor (MAF) were also used to stimulate the cells for NO production. The effect of VHSV on NO production depends on the response to LPS alone. When a low multiplicity of infection was used $\left(1.78 \times 10^{-3}\right)$, the NO production in response to LPS in LPS-responsive macrophages was significantly decreased. However, LPS-nonresponsive macrophage cultures produced NO when a combination of LPS and VHSV was used. In the case of a higher VHSV multiplicity of infection (1.78), no significant change was observed in LPSnon-responsive animals. Combinations of LPS with TNF- $\alpha$, LPS with MAF, and TNF- $\alpha$ with MAF were used to induce NO production in LPS-non-responsive macrophages. In all these cases, VHSV suppressed NO production, although at a significant level only when a combination of TNF- $\alpha$ and MAF was used for the induction of NO.
\end{abstract}

KEY WORDS: Nitric oxide · Lipopolysacharide · Macrophage · Viral hemorrhagic virus · Turbot Scophthalmus maximus · Macrophage activating factor · Tumor necrosis factor $\alpha$

\section{INTRODUCTION}

Macrophages inactivate microorganisms by different mechanisms. This microbicidal activity is, in some cases, mediated by the secretion of reactive nitrogen intermediates (Nathan \& Hibbs 1991). The molecules produced are nitrite, nitrate and nitric oxide (NO) (Marletta et al. 1988). Macrophage NO is produced via induction of inducible nitric oxide synthase (iNOS) in response to cytokines, bacterial lipopolysacharide (LPS) or parasites (Nathan 1992). NO produced by

${ }^{*}$ Corresponding author. E-mail: virus@iim.csic.es macrophages is known to play an important role in cellular defenses against specific viral infections (Croen 1993, Pertile et al. 1996, Lin et al. 1997, Tafalla et al. 1999), although there are other viruses against which NO has no effect (Kreil \& Eibl 1996, LópezGuerrero \& Carrasco 1998). Moreover, in many cases, NO mediates the viral pathogenesis in vivo (Kreil \& Eibl 1996, Adler et al. 1997). This pathogenesis may in some cases be mediated through the suppression of $\mathrm{T}$ lymphocyte proliferation, a role that has been recently attributed to NO (Allione et al. 1999).

In fish, NO production has been observed in goldfish Carassius auratus and catfish Clarias gariepinus macrophages using LPS induction (Neumann et al. 
1995, Yin et al. 1997). In the case of gilthead seabream, Sparus aurata L., induction of NO production required both macrophage-activating factor (MAF) and LPS (Mulero \& Meseguer 1998). In the turbot Scophthalmus maximus, only about one-third of macrophage cultures $(30.2 \%)$ were significantly stimulated to produce NO by LPS alone (LPS-responsive macrophages), whereas others required a combination of LPS and tumor necrosis factor $\alpha$ (TNF- $\alpha$ ), supernatants with MAF activity or supernatants with interferon (IFN)- $\alpha \beta$ activity (LPS-non-responsive macrophages) (Tafalla \& Novoa 2000). In the presence of LPS, responsive macrophages generate NO more than 2 times higher than non-stimulated controls, while the non-responsive macrophages, in the presence of LPS alone, do not generate NO concentrations significantly higher than non-stimulated controls. A combination of TNF- $\alpha$ and supernatants with MAF activity also stimulated NO production in LPS-nonresponsive macrophages.

Analysis of the effects of viral hemorrhagic septicemia virus (VHSV) on NO production is of particular interest since this virus is known to replicate in macrophages (Estepa et al. 1992, Tafalla et al. 1998). VHSV is one of the most devastating viruses in aquaculture, producing great losses in fish production. In recent years, cultured and wild populations of turbot Scophthalmus maximus have experienced significant mortalities due to VHSV outbreaks (Schlotfeldt et al. 1991, Ross et al. 1994). VHSV is known to replicate in turbot kidney macrophages; however, secretion of oxygen dependent radicals (respiratory burst activity) is not significantly affected by in vitro infection with the virus (Tafalla et al. 1998).

We have studied the effect of VHSV in vitro infection on turbot macrophage LPS induced NO production in LPS-responsive macrophages, as well as on NO induced by combinations of LPS, TNF- $\alpha$ or MAF in LPS-non-responsive macrophages. We studied the effect of these stimuli on VHSV replication to determine whether alterations in the NO production by the virus could be correlated with changes in the susceptibility of macrophages to VHSV due to these substances used for NO induction.

\section{MATERIALS AND METHODS}

Fish. Turbot Scophthalmus maximus of 40 to $60 \mathrm{~g}$ were obtained from a commercial fish farm. The animals were acclimatized to laboratory conditions for $3 \mathrm{wk}$, maintained at $18^{\circ} \mathrm{C}$ and fed daily with a commercial diet (Trow, Burgos, Spain). The health status of the animals was checked daily, and they never presented clinical symptoms and none died.
Virus and cell lines. VHSV (strain 0771) was used and propagated in TV-1, a fibroblastic cell line derived from turbot Scophthalmus maximus (FernandezPuentes et al. 1993), cultured at $18^{\circ} \mathrm{C}$ in Eagle's minimal essential medium with Earle's salts (MEM, Gibco) supplemented with $10 \%$ fetal calf serum (FCS, Gibco), containing $100 \mathrm{IU}$ of penicillin and $100 \mu \mathrm{g}$ of streptomycin $\mathrm{ml}^{-1}$. The virus was inoculated on TV-1 grown in MEM with antibiotics and $2 \% \mathrm{FCS}$ at $14^{\circ} \mathrm{C}$. When the cytopathic effect was complete, the supernatant was harvested and centrifuged to eliminate cell debris. Clarified supernatants were used for the experiments. The virus stock was titrated according to Reed \& Muench (1938) in TV-1 96 well plates.

Isolation of head kidney macrophages. Macrophages were isolated following the method described by Chung \& Secombes (1988). The anterior kidney was removed aseptically and passed through a $100 \mu \mathrm{m}$ nylon mesh using Leibovitz medium (L-15, Gibco) supplemented with penicillin (100 IU ml-1), streptomycin $\left(100 \mu \mathrm{g} \mathrm{ml}^{-1}\right)$, heparin $\left(10\right.$ units $\left.\mathrm{ml}^{-1}\right)$ and $2 \% \mathrm{FCS}$. The resulting cell suspension was placed on a $34 / 51 \%$ Percoll density gradient. The gradients were centrifuged at $500 \times g$ for $30 \mathrm{~min}$ at $4^{\circ} \mathrm{C}$. The interface cells were collected and washed twice at $500 \times g$ for 5 min in L-15 containing $0.1 \%$ FCS. The viable cell concentration was determined by trypan blue exclusion. Cells were resuspended in L-15 with $0.1 \%$ FCS and dispensed into 96 well plates at a concentration of $1 \times 10^{6}$ cells $\mathrm{ml}^{-1}\left(1 \times 10^{5}\right.$ cells well $\left.^{-1}\right)$. An aliquot of the macrophage cultures was placed on a slide and stained with Hemacolor (Merck, Darmstadt, Germany). The percentage of macrophages (always higher than $80 \%$ ) was determined by means of size, shape and staining properties. After $3 \mathrm{~h}$, the non-adherent cells were removed by washing and the resulting monolayers were exposed to the different treatments or maintained with L-15 with $5 \%$ FCS in the case of controls.

Production of MAF from turbot kidney leucocytes. Supernatants containing turbot MAF were prepared as described by Graham \& Secombes (1988) by stimulation with phorbol myristate acetate $\left(5 \mathrm{ng} \mathrm{ml}^{-1}\right)$ and concavalin A $\left(10 \mu \mathrm{g} \mathrm{ml}^{-1}\right)$. Supernatants were tested for activity on the respiratory burst of macrophages, assayed through the reduction of ferricytochrome $\mathrm{C}$ by the released superoxide, following stimulation of the

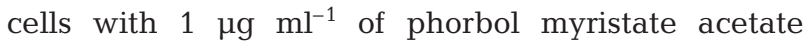
(Sigma) (Novoa et al. 1996). The supernatants were frozen at $-80^{\circ} \mathrm{C}$ until used. The working dilutions of supernatants with known MAF activity were the dilutions 1:8 and 1:16 in L-15 with 5\% FCS.

Effect of VHSV on macrophage NO production. To study the effect of VHSV in vitro infection on LPSinduced nitrite production, macrophages were incubated with different concentrations of Escherichia coli 
serotype O111:B4 LPS (Sigma), known to induce NO production in LPS-responsive macrophages $(0.1$ and $0.01 \mu \mathrm{g} \mathrm{ml}^{-1}$ ), and 2 doses of VHSV (multiplicity of infection [MOI] of $1.78 \times 10^{-3}$ and 1.78 ) in L-15 with $5 \%$ FCS, which were added simultaneously to the cultures. The LPS doses used have been determined to be optimal for stimulation of turbot macrophages (Tafalla \& Novoa 2000). The response to the different doses of LPS by the various turbot was used to characterize their macrophages as LPS-responsive or non-responsive. Non-infected controls and controls without LPS were also included. To assure that iNOS activity was being measured, the iNOS inhibitor $N^{\omega}$-nitro-L-arginine methyl ester (L-NAME; Sigma) was added in some experiments at a final concentration of $5 \mathrm{mM}$ (Tafalla \& Novoa 2000). After $72 \mathrm{~h}$ of incubation at $14^{\circ} \mathrm{C}$, the nitrite production was assayed in the cultures by the Griess reaction (Green et al. 1982) using a modification of the method described by Neumann et al. (1995). The Griess reaction quantifies the nitrite content of the macrophages supernatants, since NO is an unstable molecule and degrades to nitrite and nitrate. After incubation of macrophages at $18^{\circ} \mathrm{C}, 50 \mu \mathrm{l}$ of the macrophage supernatants was removed from individual wells and placed in a separate 96 well plate. One hundred microliters of $1 \%$ sulfanilamide (Sigma) in $2.5 \%$ phosphoric acid was added to each well, followed by $100 \mu \mathrm{l}$ of $0.1 \% \mathrm{~N}$-naphthyl-ethylenediamine (Sigma) in $2.5 \%$ phosphoric acid. Optical density was determined using a multiscan spectrophotometer (Labsystems, Helsinki) at $540 \mathrm{~nm}$. The molar concentration of nitrite in the sample was determined from standard curves generated using known concentrations of sodium nitrite.

We also determined whether VHSV altered the response of turbot LPS-non-responsive macrophages stimulated for NO production with combinations of stimuli. The optimal concentrations for NO induction of the different stimuli used had been previously determined (Tafalla \& Novoa 2000). Macrophages isolated as described above were infected with the low VHSV dose (MOI $1.78 \times 10^{-3}$ ) and immediately the different treatments were added: LPS $\left(0.01 \mu \mathrm{g} \mathrm{ml}^{-1}\right)$ and human recombinant TNF- $\alpha$ (Sigma, $\left.10 \mathrm{ng} \mathrm{ml}^{-1}\right)$, LPS $(0.01 \mu \mathrm{g}$ $\mathrm{ml}^{-1}$ ) and MAF-containing supernatants (dilution 1:8) or a combination of MAF (dilution 1:8) and TNF- $\alpha$ $\left(10 \mathrm{ng} \mathrm{ml}^{-1}\right)$. After 3 and $5 \mathrm{~d}$ of incubation, the NO production was assayed as described above.

Determination of VHSV titers. To determine whether the different cytokines or combinations of cytokines altered the susceptibility of turbot macrophages to VHSV, the viral replication (viral titer) was assayed $72 \mathrm{~h}$ after infection with VHSV (MOI $1.78 \times$ $\left.10^{-3}\right)$. The experiments were conducted as described above and, after $72 \mathrm{~h}$ at $14^{\circ} \mathrm{C}$, clarified supernatants were collected for titration. The virus present in the supernatants was titrated in TV-1 96 well plates as described before and the $\log \mathrm{TCID}_{50}$ was calculated.

Statistical analysis. The data were compared using Student's $t$-test. Results are expressed as mean \pm standard deviation and differences were considered statistically significant at $p<0.05$. All treatments were assayed by triplicate in each fish.

\section{RESULTS}

\section{Effect of VHSV on NO production induced by LPS}

To classify the macrophages as responsive or nonresponsive, in each experiment different concentrations of LPS were added to some wells and the NO production was determined in parallel in order to determine whether macrophages responded to LPS alone (data not shown). The effect of the low VHSV dose (MOI $1.78 \times 10^{-3}$ ) on the macrophage response to LPS in both types of macrophages is shown in Fig. 1A,B. Fig. 1A shows the effect of the virus on those
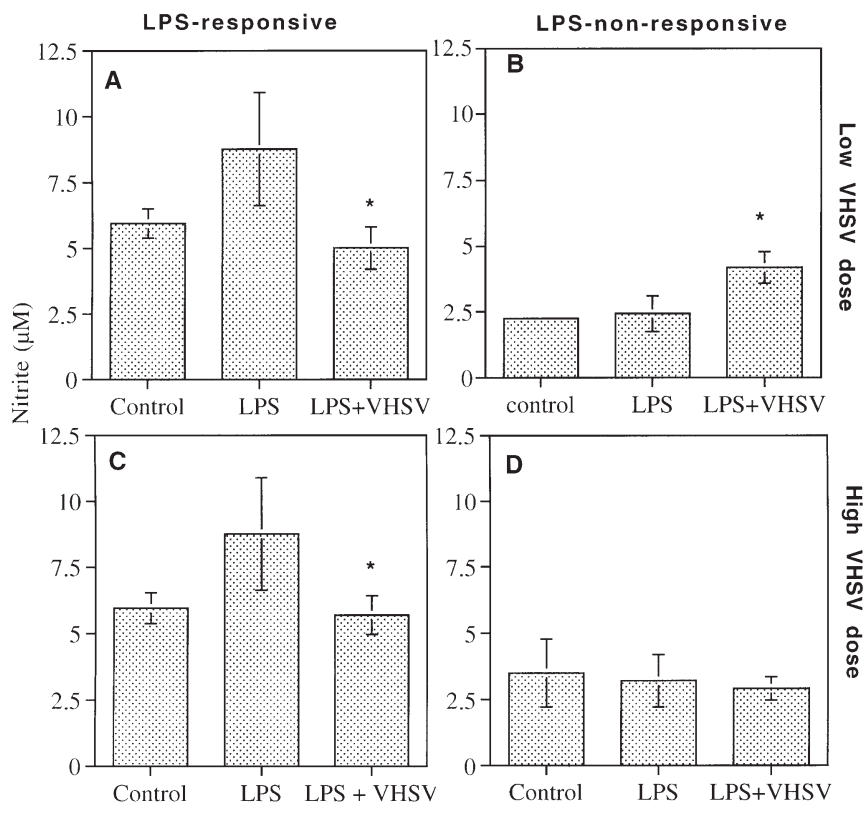

Fig. 1. Effect of the low viral hemorrhagic septicemia virus (VHSV) dose (multiplicity of infection [MOI] $1.78 \times 10^{-3}$ ) on nitric oxide (NO) production by lipopolysaccharide (LPS)responsive (A) $(n=5)$ and LPS-non-responsive macrophages (B) $(\mathrm{n}=3)$. Cells were incubated at $14^{\circ} \mathrm{C}$ for $72 \mathrm{~h}$. LPS was added at a concentration of $0.01 \mu \mathrm{g} \mathrm{ml}^{-1}$. The effects of the high VHSV dose (MOI 1.78) on NO production by LPSresponsive $(\mathrm{C})(\mathrm{n}=5)$ and LPS-non-responsive macrophages (D) $(n=3)$ are also shown. LPS was also added at a concentration of $0.01 \mathrm{\mu g} \mathrm{ml}^{-1}$. Data are expressed as mean concentration of nitrite $(\mu \mathrm{M}) .{ }^{*} \mathrm{NO}$ production significantly differentfrom that of macrophages treated with LPS alone $(p<0.05)$ 
animals that responded to LPS. These macrophages were significantly stimulated for NO production with $0.01 \mathrm{~g} \mathrm{~m} \mathrm{ml}^{-1}$ of LPS. In this case, the virus in combination with LPS decreased the production of NO compared with that observed when macrophages were incubated with LPS alone. However, macrophages that did not respond to LPS (Fig. 1B) when incubated with the virus and LPS had a significantly increased NO production upon infection. These 2 responses were always observed when the experiments were repeated.

In LPS-responsive macrophages, the effect of the high VHSV dose (MOI 1.78) was the same as that observed with the low viral dose (Fig. 1C). However, when the high dose was used, no significant effect on NO production was observed in LPS-non-responsive macrophages (Fig. 1D).

In all cases, the VHSV alone never significantly altered the NO production of macrophages after $72 \mathrm{~h}$ of incubation.

\section{Effect of L-NAME on NO production}

To determine whether the iNOS activity was really being measured, L-NAME was added. The results obtained when L-NAME was added to LPS-nonresponsive macrophage cultures are shown in Fig. 2. The inhibitor significantly decreased the nitrite production observed with and without LPS. In this experiment, again the nitrite production observed with LPS and the low VHSV dose was significantly higher than that observed with LPS alone, and L-NAME partially reversed this NO induction. This decrease was only significant when individual data and not mean values were compared. In the case of LPS-responsive macrophage cultures, the inhibitor significantly decreased the LPS-induced nitrite production (Fig. 3).

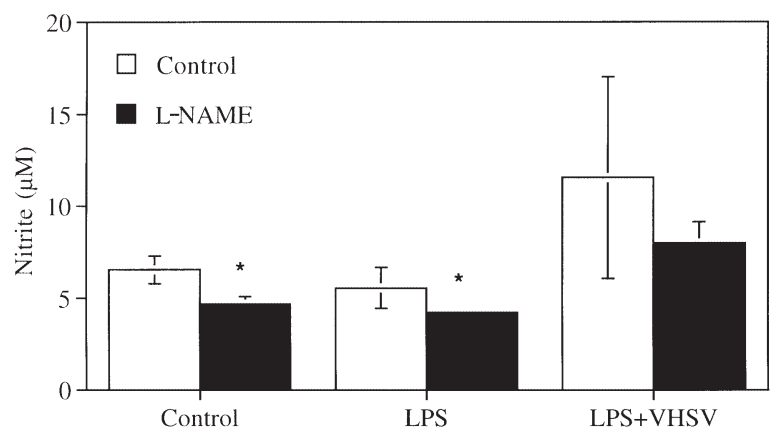

Fig. 2. Effect of $N^{\omega}$-nitro-L-arginine methyl ester (L-NAME; $5 \mathrm{mM}$ ) on the NO production of LPS-non-responsive macrophage cultures. Data are expressed as mean nitrite $(\mu \mathrm{M})$ produced by 3 fish. ${ }^{*}$ NO production significantly lower than that in control macrophages not treated with L-NAME $(p<0.05)$

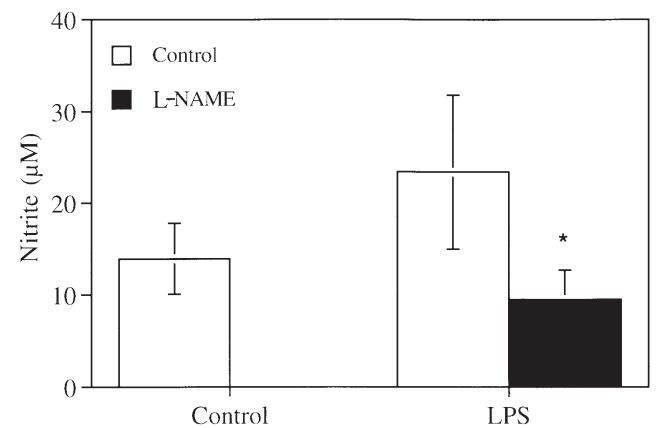

Fig. 3. Effect of L-NAME ( $5 \mathrm{mM})$ on the NO production of LPS-responsive macrophage cultures. Data are expressed as mean nitrite $(\mu \mathrm{M})$ produced by 3 fish. ${ }^{*}$ NO production significantly lower than that in control macrophages not treated with L-NAME $(p<0.05)$

\section{Effect of VHSV on the response to combinations of stimuli in LPS-non-responsive macrophages}

The effect of VHSV (low MOI) on NO induced by LPS combined with TNF- $\alpha$ or with MAF, and with TNF- $\alpha$ combined with MAF was assayed. The results observed after $3 \mathrm{~d}$ of incubation are shown in Fig. 4A. All the combinations induced NO production in individual macrophage cultures; however, when mean values were compared, only TNF- $\alpha$ and MAF were found to significantly induce NO. In this case, VHSV significantly suppressed this NO induction. The same response was observed after $5 \mathrm{~d}$ of incubation (Fig. 4B). In the non-infected controls, after $3 \mathrm{~d}$ of incubation, the NO produced in response to TNF- $\alpha$ and MAF was significantly higher than that observed with LPS and TNF- $\alpha$; however, after $5 \mathrm{~d}$ of incubation, the NO production observed with the different combinations was not significantly different.

\section{Effect of the different stimuli on viral replication in turbot macrophages}

To determine whether the differences in NO production induced by the various inducers in the presence of VHSV were due to an altered replication of VHSV in macrophages treated with these substances, the viral replication with and without stimulation was determined. No significant differences were found between the VHSV titer obtained in macrophages treated with any of the substances or combinations and the viral titer observed with VHSV alone. After $3 \mathrm{~d}$ of incubation, the $\log \mathrm{TCID}_{50} \mathrm{ml}^{-1}$ in the presence of LPS was $3.8 \pm 0.37$ and $4 \pm 0.5$ in cultures treated with only VHSV. In all cases, the virus replicated, since the viral titer increased with time. 

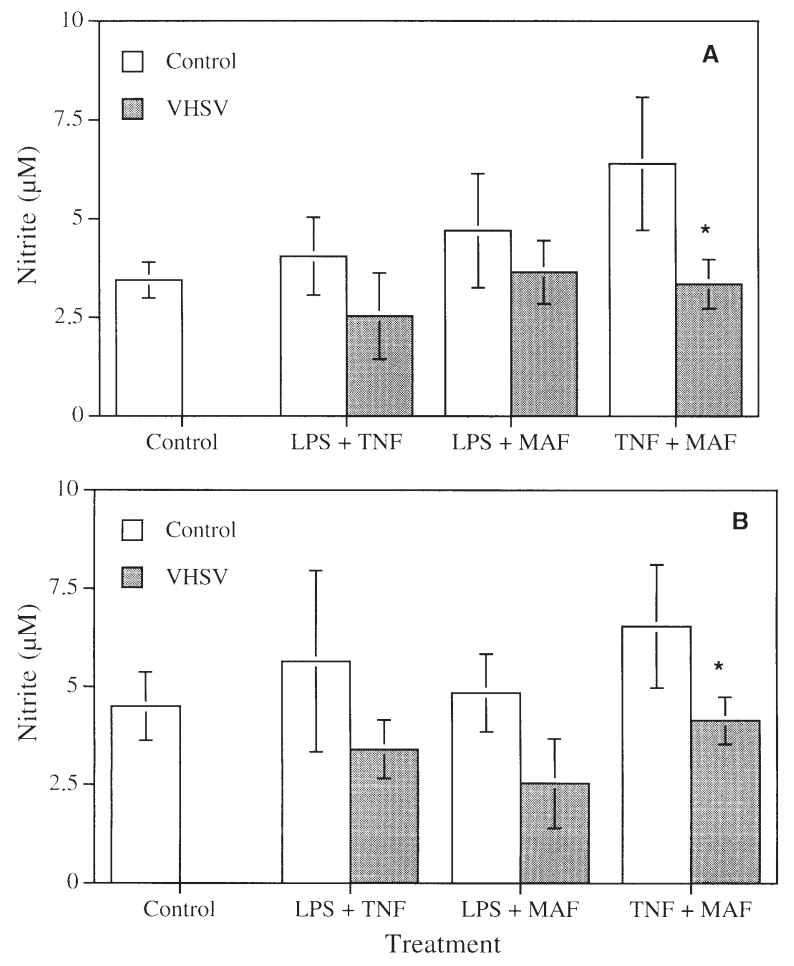

Fig. 4. Effect of VHSV (MOI $1.78 \times 10^{-3}$ ) in vitro infection on the NO production induced by LPS and tumor necrosis factor $\alpha$ (TNF- $\alpha$ ), LPS and macrophage-activating factor (MAF) or TNF- $\alpha$ and MAF after (A) 3 or (B) $5 \mathrm{~d}$ of incubation. Data are expressed as mean nitrite $(\mu \mathrm{M}) . \mathrm{n}=4 .{ }^{*} \mathrm{NO}$ production significantly lower than that observed in the respective stimulated control not infected with VHSV

\section{DISCUSSION}

It has been shown that VHSV replicates in turbot macrophages, although only a small percentage of the cells support active replication (1.7\%) (Tafalla et al. 1998). Concerning turbot macrophage NO production induced by LPS, we have previously found that macrophages isolated from individual turbot can exhibit 2 different responses to LPS: about one-third of the animals yielded macrophages that produced $\mathrm{NO}$ in response to a single stimulation with LPS $(30.2 \%)$ (LPS-responsive macrophages) whereas the others required a combination of LPS and TNF- $\alpha$, IFN- $\gamma$ or IFN- $\alpha \beta$ (LPS-non-responsive macrophages) (Tafalla \& Novoa 2000). Some authors reported that, in mice, macrophages do not respond to LPS alone but need a previous exposure to IFN- $\gamma$ (Kreil \& Eibl 1995) but other authors have reported stimulation of NO production with LPS alone (López-Collazo et al. 1998). In the case of bovine macrophages and neutrophils, it has been shown that, in some individuals, both cell types respond to individual stimulants like IFN- $\gamma$ or TNF- $\alpha$, while other individuals require a combination of IFN- $\gamma$ and TNF- $\alpha$ to produce NO (Goff et al. 1996).

In turbot, we have found that when macrophages are infected in vitro with a low VHSV dose, responses are strikingly different in LPS-responsive versus LPS-nonresponsive macrophages. In LPS-responsive macrophages, the virus caused a decrease in NO production, whereas in LPS-non-responsive macrophages a significant increase in NO production with a combination of LPS and VHSV was observed. When combinations of stimuli were used to induce $\mathrm{NO}$, we also found a suppression of the NO production in macrophages infected with VHSV. This suppression was significant when TNF- $\alpha$ and MAF were combined, probably because this was the combination that induced the higher NO production. This suppression was observed after 3 and $5 \mathrm{~d}$ of incubation.

The suppression of NO production seen in both cases (LPS-responsive macrophages stimulated with LPS and LPS-non-responsive macrophages stimulated with a combination of MAF and TNF- $\alpha$ ) could be due to intrinsic interactions between macrophages and the virus that usually alter macrophage functions (Morahan et al. 1985). We determined that these substances used in NO induction do not alter the replication of VHSV in turbot macrophages (viral titer after $3 \mathrm{~d}$ ), so this decrease was not due to an increased susceptibility but rather to an interaction between the macrophages and the virus. It is known that viral infections usually produce a depression of macrophage functions, even when only a subpopulation is infected, although no effect and even enhanced activity have also been reported (Lussenhop et al. 1982, Mogesen 1982). Macrophages are known to be heterogeneous for viral infection, as frequently a subpopulation is infected and the outcome of the infection is dependent on a great number of host-related factors, including the state of differentiation or activation of the immunocompetent cells or even genetic determinants (Morahan et al. 1985).

The up-regulation of NO production observed in the case of the LPS-non-responsive macrophages in the presence of VHSV could be explained as a result of an antiviral defense mechanism being triggered in macrophages, since NO is known to have an antiviral effect on many viruses (Croen 1993, Pertile et al. 1996, Lin et al. 1997), including antiviral activity toward VHSV (Tafalla et al. 1999). It is known that viral infection of macrophages leads to the production of IFN- $\alpha \beta$ (de Maeyer \& de Maeyer-Guignard 1991) and that in some cases this IFN has a priming effect on macrophages for further LPS activation (Fujihara et al. 1994, Kreil \& Eibl 1995). In mice, it was determined that macrophages were not stimulated to produce NO with LPS alone and required another activating factor, while virus-infected 
macrophages produced NO in response to LPS alone due to the secretion of IFN- $\alpha \beta$ in infected macrophages (Kreil \& Eibl 1995). In turbot, it has been previously determined that a combination of LPS and IFN- $\alpha \beta$ significantly stimulates otherwise non-responsive macrophages for NO production (Tafalla \& Novoa 2000). It could be possible that these LPS-non-responsive macrophages produce IFN- $\alpha \beta$ in response to the virus. This IFN- $\alpha \beta$ secretion would also make them less susceptible to VHSV infection, since IFN- $\alpha \beta$ is known to have antiviral activity (de Maeyer \& de MaeyerGuignard 1991). It is known that constitutive viral resistance is usually mediated through high NO production (López-Guerrero et al. 1997). This up-regulation in response to VHSV disappeared when the high VHSV was used, suggesting that if this response constitutes some type of antiviral activity, it is significant only at low viral doses.

In conclusion, turbot macrophages that differ in their response to LPS also differ in their response to VHSV infection, which is probably related to the outcome of the infection. Whenever NO production was induced, VHSV suppressed this induction, whereas when NO was not induced, VHSV acted as a second stimulating signal or co-stimulator to induce NO production.

Acknowledgements. This research was supported by grant MAR 96-1775 from the Comisión Interministerial de Ciencia y Tecnología (CICYT) (Spain). C.T. acknowledges the Fundación Ramón Areces for a research fellowship. We also want to thank Begoña Villaverde, María Jesús Pérez Garabito, Dolores Dominguez and José Ramón Caldas for their technical assistance and cooperation.

\section{LITERATURE CITED}

Adler H, Beland JL, Del-Pan NC, Kobzik L, Brewer JP, Martin TR, Rimm IJ (1997) Suppression of herpes simplex virus type 1 (HSV-1)-induced pneumonia in mice by inhibition of inducible nitric oxide synthase (iNOS, NOS2). J Exp Med 185:1533-1540

Allione A, Bernabei P, Bosticardo M, Arioti S, Forni G, Novelli F (1999) Nitric oxide suppresses human T lymphocyte proliferation through IFN-dependent and IFN-independent induction of apoptosis. J Immunol 163:4182-4191

Chung S, Secombes CJ (1988) Analysis of events occurring within teleost macrophages during the respiratory burst. Comp Biochem Physiol 89B:539-544

Croen KD (1993) Evidence for antiviral effect of nitric oxide: inhibition of herpes simplex virus type 1 replication. J Clin Invest 91:2446-2452

de Maeyer E, de Maeyer-Guignard J (1991) In: Thomson A (ed) The cytokine handbook, 1991. Academic Press, London, p 215-239

Estepa A, Frias D, Coll JM (1992) Susceptibility of trout kidney macrophages to viral haemorrhagic septicemia virus. Viral Immunol 4:283-292

Fernandez-Puentes C, Novoa B, Figueras A (1993) A new fish cell line derived from turbot (Scophthalmus maximus) TV-1. Bull Eur Assoc Fish Pathol 13:94-96
Fujihara M, Ito N, Pace JL, Watanabe Y, Russell SW, Sukuki T (1994) Role of interferon- $\beta$ in lipopolysaccharide-triggered activation of the inducible nitric-oxide synthase gene in a mouse macrophage cell line J774. J Biol Chem 269: 12773-12778

Goff WL, Johnson WC, Wyatt CR, Cluff CW (1996) Assessment of bovine mononuclear phagocytes and neutrophils for induced L-arginine-dependent nitric oxide production. Vet Immunol Immunopathol 55:45-62

Graham S, Secombes CJ (1988) The production of macrophage-activating factor from rainbow trout Salmo gairdneri leucocytes. Immunology 65:293-297

Green LC, Wagner DA, Glogowski J, Skipper PL, Wishnok JS, Tannenbaum S (1982) Analysis of nitrate, nitrite, and (15N) nitrate in biological fluids. Anal Biochem 126: 131-138

Kreil KR, Eibl MM (1995) Viral infection of macrophages profoundly alters requirements for induction of nitric oxide synthesis. Virology 212:174-178

Kreil KR, Eibl MM (1996) Nitric oxide and viral infection: no antiviral activity against a flavovirus in vitro, and evidence for contribution to pathogenesis in experimental infection in vivo. Virology 219:304-306

Lin YL, Huang YL, Ma SH, Yeh CT, Chiou SY, Chen LK, Liao CL (1997) Inhibition of Japanese encephalitis virus infection by nitric oxide: antiviral effect of nitric oxide on RNA virus replication. J Virol 71:5227-5235

López-Collazo E, Hortelano S, Rojas A, Boscá L (1998) Triggering of peritoneal macrophages with INF- $\alpha / \beta$ attenuates the expression of inducible nitric oxide synthase through a decrease in NF- $\kappa B$ activation. J Immunol 160:2889-2895

López-Guerrero JA, Carrasco L (1998) Effect of nitric oxide on poliovirus infection of two human cell lines. J Virol 72: $2538-2540$

López-Guerrero JA, Rayet B, Tuynder M, Rommelaere J, Dinsart C (1997) Constitutive activation of U937 promonocytic cell clones selected for their resistance to parvovirus $\mathrm{H}-1$ infection. Blood 89:1642-1653

Lussenhop N, Holmes B, Cafruny WA, Plagemann PGW (1982) Acute infection of mice with lactate dehydrogenase-elevating virus enhances $\mathrm{FC}_{\mathrm{C}}$ and complement receptor activity of peritoneal macrophages. J Gen Virol 61: $25-32$

Marletta MA, Yoon PY, Iyengar R, Leaf CD, Wishnok JS (1988) Macrophage oxidation of L-arginine to nitrite and nitrate: nitric oxide is the intermediate. Biochemistry 27 : 8706-8711

Mogesen SC (1982) Viral interference with the function of phagocytic cells. In: FEMS Symposium of Bacterial and Viral Inhibition and Modulation of Host Defense 1982. Academic Press, New York

Morahan PS, Connor JR, Leary KR (1985) Viruses and the versatile macrophage. Br Medical Bull 41:15-21

Mulero V, Meseguer J (1998) Functional characterisation of a macrophage-activating factor produced by leucocytes of gilthead seabream (Sparus aurata L.). Fish Shellfish Immunol 8:143-156

Nathan C (1992) Nitric oxide as a secretory product of mammalian cells. FASEB J 6:3051-3064

Nathan CF, Hibbs JB Jr (1991) Role of nitric oxide synthesis in macrophage antimicrobial activity. Curr Opin Immunol 3: $65-70$

Neumann NF, Fagan D, Belosevic M (1995) Macrophage activating factor(s) secreted by mitogen stimulated goldfish kidney leucocytes synergize with bacterial lipopolysaccharide to induce nitric oxide production in teleost macrophages. Dev Comp Immunol 19:473-482 
Novoa B, Figueras A, Ashton I, Secombes CJ (1996) In vitro studies on the regulation of rainbow trout (Oncorhynchus mykiss) macrophage respiratory burst activity. Dev Comp Immunol 20:207-216

Pertile TL, Karaca K, Sharma JM, Walser MM (1996) An antiviral effect of nitric oxide: inhibition of reovirus replication. Avian Dis 40:342-348

Reed LJ, Muench H (1938) A simple method of estimating fifty per cent end-points. Am J Hyg 27:493-497

Ross K, McCarthy U, Huntly PJ, Wood BP, Stuart D, Rough EI, Smail DA, Bruno DA (1994) An outbreak of viral haemorrhagic septicemia (VHSV) in turbot (Scophthalmus maximus) in Scotland. Bull Eur Assoc Fish Pathol 14:213-214

Schlotfeldt HJ, Anhe W, Vestergard-Jorgensen PE (1991)

Occurrence of viral haemorrhagic septicemia in turbot (Scophthalmus maximus). A natural outbreak. Bull Eur

Editorial responsibility: Jo-Ann Leong,

Corvallis, Oregon, USA
Assoc Fish Pathol 11:105-107

Tafalla C, Novoa B (2000) Requirements for nitric oxide production by turbot (Scophthalmus maximus) head kidney macrophages. Dev Comp Immunol 24:623-631

Tafalla C, Figueras A, Novoa B (1998) In vitro interaction of viral haemorrhagic septicaemia virus and leucocytes from trout (Oncorhynchus mykiss) and turbot (Scophthalmus maximus). Vet Immunol Immunopathol 62:359-366

Tafalla C, Figueras A, Novoa B (1999) Role of nitric oxide on the replication of viral haemorrhagic septicemia virus (VHSV), a fish rhabdovirus. Vet Immunol Immunopathol 72:249-256

Yin Z, Lam TJ, Sin YM (1997) Cytokine-mediated antimicrobial immune response of catfish, Clarias gariepinus, as a defense against Aeromonas hydrophila. Fish Shellfish Immunol 7:93-104

Submitted: August 7, 2000; Accepted: August 2, 2001

Proofs received from author(s): October 30, 2001 